\title{
Reversible superior vena caval syndrome due to tuberculosis
}

\author{
RICHARD G HARBECKE, ${ }^{1}$ DONALD P SCHLUETER, AND DAVID Y ROSENZWEIG \\ From the Pulmonary Medicine Section, Department of Medicine, Medical College of Wisconsin, \\ Milwaukee, Wisconsin, USA
}

Obstruction of the superior vena cava is usually caused by neoplasia; however, benign diseases cause between 3 and $26 \%$ of the reported cases (Lokich and Goodman, 1975; Urschel and Paulson, 1966). We describe a superior vena caval syndrome that resolved with chemotherapy for tuberculosis.

\section{Case report}

A 36-year-old man was admitted on 21 January 1976 after he lacerated the flexor tendon of his right index finger. He had a prominent superficial venous pattern on his chest and digital clubbing. A chest radiograph showed a large hilar mass (see figure). He had noticed distended veins on his neck and chest for the past year, but denied any history of tuberculosis or respiratory symptoms. He had smoked one packet of cigarettes a day, stopping five years before admission.

Sputa for cytology and smear for acid-fast bacilli (AFB) were negative on several occasions. The 5 TU tuberculin skin test was positive. His superior venacavogram showed complete occlusion of the superior

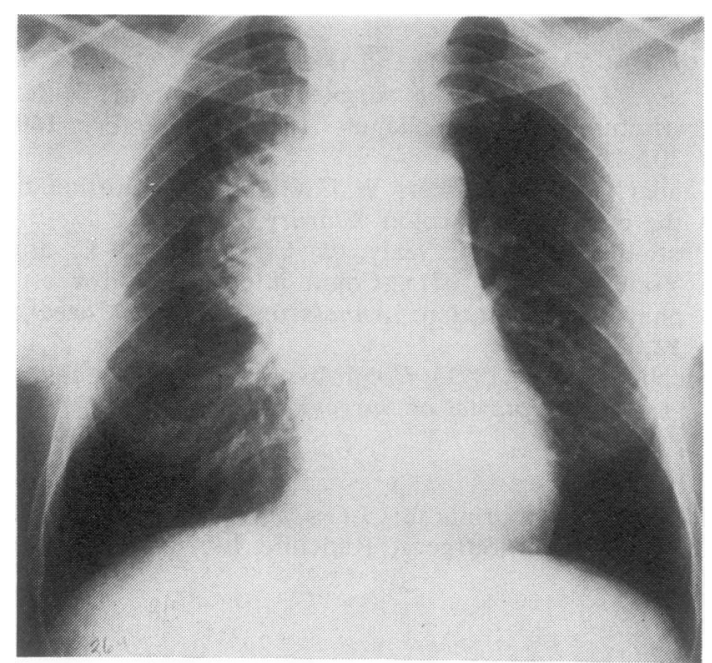

Routine admission chest radiograph showing right hilar mass.

'Wisconsin Lung Association Fellow in Pulmonary Medicine. Present address: Milwaukee Medical Clinic, SC, Milwaukee, Wisconsin 53209. vena cava with large collateral veins. On bron- $\vec{\circ}$ choscopy, an erythematous trachea and right main $\rightleftharpoons$ bronchus were seen, but specimens for cytology and $x$ smear for AFB and fungi were negative. Mediastino- $\omega$ scopy showed a hard right paratracheal mass. Biopsies, $\dot{\omega}$ however, failed to show any tumour, granulomas, or $\stackrel{A}{A}$ AFB. A right axillary abscess was also drained. The $\vec{O}$ patient was then discharged to await cultures before 음 proceeding with thoracotomy.

The scar from the axillary abscess was reopened on 10 February 1976 and caseating granulomas and a single AFB were seen in an excised node. Several sputa were then reported to be growing Mycobacterium $\vec{\varphi}$ tuberculosis. Treatment began, using isoniazid, etham- oै butol, and rifampicin. At this time the patient's old records became available and showed that a right suprascapular abscess had been drained in 1969 and grew $M$ tuberculosis, but that the patient had been lost to follow-up before treatment began. Slight widening of the upper mediastinum was noted on the chest radiograph in 1969.

Because of the possibility of a coexistent neoplasm with reactivation of his tuberculosis, a limited thoracotomy was performed after six weeks of chemotherapy. Biopsies showed multiple non-caseating granulomas but no AFB on smear or culture, and no tumour. After eight weeks of chemotherapy the venous obstruction on his neck and chest had resolved. He has done well since with continued improvement in his chest radiograph and freedom from symptoms of 3 . superior vena caval obstruction.

\section{Discussion}

Tumours accounted for only $15-37 \%$ of cases of $\supset$ superior vena caval obstruction reported in the early part of this century (Ochsner and Dixon, 1935-6). N More recent reports have estimated that up to $97 \%$ of $\sigma$ the cases of this syndrome are due to neoplasms, $N$ mainly bronchogenic carcinoma (Lokich and Good- N man, 1975). Tuberculosis accounted for $11.4 \%$ of the $\omega$ cases reported between 1904 and 1911. This declined to $5.4 \%$ between 1934 and 1946, paralleling the improvement in prevention and treatment of tuberculosis. The table shows the aetiology of superior vena caval ? obstruction in 276 cases reported in the English literature between 1967 and 1976. Tuberculosis was diagnosed in only one case $(0.3 \%)$. Most of the credit for this decrease is attributable to improvement in chemotherapy for tuberculosis, but greater diagnostic specificity may also be a factor. 
Aetiology of 276 cases of superior vena caval obstruction reported between 1967-76. (Percentages in parentheses)

\begin{tabular}{|c|c|c|c|}
\hline \multicolumn{2}{|l|}{ Malignant $203(73 \cdot 6)$} & \multicolumn{2}{|l|}{ Benign 73 (26.4) } \\
\hline Bronchogenic carcinoma & $135(48 \cdot 9)$ & Histoplasmosis & $15(5 \cdot 4)$ \\
\hline $\begin{array}{l}\text { "Probable" bronchogenic } \\
\text { carcinoma }\end{array}$ & $37(13 \cdot 4)$ & $\begin{array}{l}\text { Unspecified fibrosis } \\
\text { and granulomas }\end{array}$ & $14(5 \cdot 1)$ \\
\hline $\begin{array}{l}\text { “Other” neoplasms } \\
\text { including metastatic }\end{array}$ & $31(11 \cdot 2)$ & $\begin{array}{l}\text { Goitres } \\
\text { Iatrogenic and after } \\
\quad \text { surgery } \\
\text { Aneurysms } \\
\text { Behcet's } \\
\text { Miscellaneous } \\
\text { Tuberculosis }\end{array}$ & $\begin{array}{l}12(4 \cdot 3) \\
9(3 \cdot 3) \\
8(2 \cdot 9) \\
7(2 \cdot 5) \\
7(2 \cdot 5) \\
1(0 \cdot 3)\end{array}$ \\
\hline
\end{tabular}

Benign cases of superior vena caval obstruction differ from those due to tumour in several ways. Firstly, patients with the benign syndrome usually complain of symptoms for months to years whereas those with a malignant tumour usually present with symptoms developing over several weeks. With benign disease, patients tend to be younger (usually in the 40 s) than those with cancer who are usually in the $50 \mathrm{~s}$ or $60 \mathrm{~s}$. Of patients with cancer $80 \%$ are men, whereas this predominance is absent in benign cases. Finally, the benign type of superior vena caval syndrome is compatible with a long life expectancy.

Establishing a definitive diagnosis often requires exploratory surgery as in this case. In young patients with prolonged symptoms if diagnostic studies are unrewarding a period of observation seems advisable. Surgery is controversial in cases caused by fibrosis or granuloma. Several bypass and reconstructive procedures to alleviate vena caval obstruction have been reported with mixed success (Effler and Groves, 1962).
Current opinion, however, favours non-intervention since this syndrome has a remarkably good long-term prognosis and functionally important collaterals may be ligated during surgery in an attempt to control bleeding (Urschel and Paulson, 1966). Diuretics, sodium restriction, and steroids have been advocated to control acute symptoms (Lokich and Goodman, 1975).

Antituberculosis chemotherapy is indicated in cases caused by tuberculosis. Owing to the paucity of recent cases, it is difficult to say what can be expected with current chemotherapy. Our patient has been symptom free for 24 months, and there has been continued shrinkage of his right hilar mass while on chemotherapy for tuberculosis. His limited thoracotomy did not include any surgical attempt to relieve the obstruction, but this may have affected his course as symptoms abated two weeks later.

\section{References}

Effler, D B, and Groves, L K (1962). Superior vena caval obstruction. Journal of Thoracic and Cardiovascular Surgery, 43, 574-584.

Lokich, J J, and Goodman, R (1975). Superior vena cava syndrome-clinical management. Journal of the American Medical Association, 231, 58-61.

Ochsner, A, and Dixon, J L (1935-6). Superior vena cava thrombosis. Journal of Thoracic Surgery, 5, $641-672$.

Urschel, H C, and Paulson, D L (1966). Superior vena caval obstruction. Diseases of the Chest, 49, 155-164.

Requests for reprints to: Donald P Schlueter, MD, Medical College of Wisconsin, 8700 West Wisconsin Avenue, Milwaukee, Wisconsin 53226. 